were participants of the 2007 National Roadside Survey of Alcohol and Drug Use by Drivers.

Results Overall, $53.3 \%$ of the cases and $8.9 \%$ of the controls had elevated blood alcohol concentrations (BAC $\geq 0.01 \mathrm{~g} / \mathrm{dL}$ ), and $6.0 \%$ of the cases and $3.0 \%$ of the controls tested positive for marijuana. Compared to drivers testing negative for both alcohol and marijuana, the estimated odds ratios of fatal crash involvement were 11.77 [95\% confidence interval (CI): 10.78, 12.84] for those testing positive for alcohol and negative for marijuana, 2.16 (95\% CI: $1.85,2.54)$ for those testing negative for alcohol and positive for marijuana, and 25.46 (95\% CI: 21.25, 30.51) for those testing positive for both alcohol and marijuana.

Conclusions Alcohol and marijuana are each associated with a significantly increased risk of fatal crash involvement. When alcohol and marijuana are used together, there exists a positive interaction effect on the risk of fatal crash involvement on the additive and the multiplicative scales.

\section{IMPACT OF COLOUR-GRADED PICTOGRAM ON MEDICINE PACKAGES TO CAUTION AGAINST THE RISK OF TRAFFIC CRASH}

1,2Ludivine Orriols, 1,2Audrey Luxcey, 1,2Benjamin Contrand, 3,4Blandine Gadegbeku ,'Bernard Delorme, ${ }^{2,6,7}$ Nicholas Moore, ${ }^{1,2}$ Emmanuel Lagarde. 'INSERM, IETO Team, ISPED, France; ${ }^{2}$ University of Bordeaux, France; ${ }^{3}$ IFSTTAR, UMR T9405, UMRESTTE, France; ${ }^{4}$ Lyon 1 University, France; ${ }^{5}$ ANSM, France; ${ }^{6}$ INSERM U657, France; ${ }^{7}$ CIC-1401, France

\subsection{6/injuryprev-2016-042156.298}

Background In France, a colour-graded pictogram is printed on the outer packaging of medicines according to their effect on driving performance, from level 1 (low risk) to level 3 (high risk). The aim of this study was to assess the impact of labelling benzodiazepines and z-hypnotics with level 2 or 3 pictograms on the risk of road traffic crash.

Methods Data from three French national databases were extracted and matched: the healthcare insurance database, police reports, and the police database of injurious crashes. Drivers involved in an injury crash in France, from July 2005 to December 2011, and identified by their national identifier were included. The study period was divided into 4 periods. The first period corresponded to a period before the colour-graded threelevel pictogram was set up. The immediately subsequent period was used to estimate the impact of the introduction of the threelevel pictogram. The two following time-periods were defined to assess any relapse in the potential impact of the pictogram. A case-control analysis comparing responsible versus non-responsible drivers was conducted.

Results 142,763 drivers were included. Exposure to benzodiazepine anxiolytics (level 2 or 3) was associated with an increased risk of being responsible for a road traffic crash during the first period of the study $(\mathrm{OR}=1.42$ [1.24-1.62]). The association disappeared in the second period and became significant again during the third and the fourth period. The risk of being responsible for a crash increased in users of z-hypnotics (level 3) across the four periods (OR from $0.97[0.81-1.17]$ to 1.32 [1.101.60]).

Conclusions Our results suggest that there has been a change in driving behaviour in benzodiazepine anxiolytic users after the implementation of the graded pictogram. However, there was a relapse immediately after. The increased risk associated with zhypnotic use despite the presence of the higher level of pictogram calls for further preventive interventions.

\section{RISK COMMUNICATION IN MEDICINES AND DRIVING USING PICTOGRAMS}

Han de Gier. The University of Groningen Research Institute of Pharmacy, Department of Pharmacotherapy and Pharmaceutical Care, Groningen, Netherlands

\subsection{6/injuryprev-2016-042156.299}

Background: Appropriate communication towards patients using driving impairing medicines about risk on fitness to drive can be supported by the use of pictograms. To evaluate and compare the effectiveness of various pictograms and warnings (e.g. a French, a Dutch and a newly developed EU rating model) in communicating risk, several experiments were conducted.

Methods: Structured interviews (experiment 1) and written questionnaires (experiment 2) were used respectively among drivers $(n=270)$ visiting one out of four community pharmacies in Groningen $(n=4)$ and patients who were drivers $(n=298)$ and starting a new treatment with a driving impairing medicine visiting community pharmacies $(\mathrm{n}=38)$ in the Netherlands, to compare various pictograms and warnings.

Results: Compared to general warnings and pictograms, the EU rating model allowed patients to better understand the potential risk estimate when using impairing medicines. Addition of a sidetext to the rating model resulted in a significantly higher estimated level of driving risk and a significant increase in intention to change driving behaviour. Age was the strongest predictor influencing participants' preference for pictograms to express a warning message and levels of impairment.

Conclusions: Implementation of the rating model in clinical practice should be considered, but factors such as age and education of patients need attention in designing and implementing new pictograms.

\section{UNLOCKING KEYS TO EFFECIVE IGNITIION INTERLOCK PROGRAMS TO REDUCE ALCOHOL IMPAIRED DRIVING}

${ }^{1}$ Gwen Bergen, Bethany West, ${ }^{2}$ Tara Casanova Powell, ${ }^{3}$ Robert Voas, Erin Sauber-Schatz, Ann Dellinger, Grant Baldwin. 'U.S. Centres for Disease Control and Prevention, National Centrefor Injury Prevention and Control, U.S.A.; ${ }^{2}$ Traffic Injury Research Foundation, Formerly During the Publication of the Study-Preusser Research Group, U.S.A.; ${ }^{3}$ Pacific Institute for Research and Evaluation, U.S.A

\subsection{6/injuryprev-2016-042156.300}

Background Ignition interlocks, when installed on vehicles of drivers convicted of alcohol-impaired driving (AID), reduce repeat arrest by $67 \%$. However post-interlock removal, recidivism (AID re-arrest) among previous interlock users equals that of AID-convicted drivers who never used interlocks. Also, the low numbers of offenders installing interlocks limits the impact. Study objectives include determining interlock program characteristics associated with increased interlock use and evaluating including alcohol treatment in the program to reduce post-interlock recidivism.

Methods To determine effective program characteristics, eight interlock program keys (e.g. requirement to instal interlocks) were identified and each rated on 1-5 scale for 28 U.S. state interlock programs. Correlation analysis between rate of interlocks in use $/ 10,000$ population, and program key rating was conducted. To evaluate treatment in the one state with a treatment program, survival analysis using Cox regression proportional hazards model was performed with post-interlock recidivism as the terminal event. The treatment group $(n=640)$ were offenders with three or more violations (two alcohol-positive start attempts 
within four hours) who completed alcohol treatment. A comparison group $(\mathrm{n}=806)$ of those with one or two violations who did not attend alcohol treatment was created by matching to the treatment group on demographic and risk factors.

Results The program keys most correlated with higher interlock rates were having a requirement to instal interlocks $(r=0.63)$, and monitoring to ensure interlocks are installed and used $(\mathrm{r}=0.56)$. Incorporating alcohol treatment into an interlock program was effective with the treatment group experiencing $32 \%$ lower recidivism following interlock removal compared with the non-treatment group.

Conclusions Strengthening program keys and incorporating treatment into interlock programs increases use of interlocks and reduces AID re-arrest.

\section{MARIJUANA USE AND EXCESS RISK OF INJURY EVENTS: FINDINGS FROM A LARGE PROSPECTIVE COHORT STUDY}

${ }^{1}$ Shanthi Ameratunga, ${ }^{1}$ Roshini Peiris-John, ${ }^{1}$ Papaarangi Reid, ${ }^{1}$ Arier Lee, ${ }^{2}$ Gordon S Smith, ${ }^{3}$ Robyn Norton. ${ }^{1}$ University of Auckland, New Zealand; ${ }^{2}$ University of Maryland, USA; ${ }^{3}$ The George Institute for Global Health, Australia

\subsection{6/injuryprev-2016-042156.301}

Background Marijuana is one of most widely used illicit substances globally. The risks of injury experienced by marijuana users relative to non-users have been poorly quantified in prospective studies. We investigated the associations between marijuana use and fatal or hospitalised injury in the New Zealand Blood Donors' Health Study.

Methods At recruitment to this prospective cohort study in 1998-1999, the 22,389 participants completed a self-report survey on demographic, personal and lifestyle questions including the frequency of marijuana use. Using unique identifiers, these data were record-linked to national mortality and hospital discharge databases to ascertain participants' injury-related admissions or deaths from recruitment to 31 December 2014. The associations between marijuana use and injury were investigated using Cox proportional hazards analyses, adjusting for sociodemographic and confounding factors.

Results At baseline, $15 \%$ of participants of the cohort reported marijuana use in the preceding 12 months. During a median follow-up period of 16.8 years, 3,693 incident injury cases $(3,651$ non-fatal and 63 fatal) occurred, the majority of which were falls (1204 incident cases) and motor vehicle crashes (779 incident cases). Compared to non-users, marijuana users were more likely to experience injuries related to self-harm (adjusted HR 2.00, 95\% CI: 1.39-2.89), assault (adjusted HR 1.83, 95\% CI: $1.27-$ 2.66), motor vehicle crashes (adjusted HR 1.35, 95\% CI: 1.091.69), or unintended cutting or piercing trauma (adjusted HR 1.76, 95\% CI: 1.31-2.35). Risk estimates were greater with more frequent use of marijuana.

Conclusions Relative to non-users, participants who used marijuana were at increased risk of most major types of injuries, with an apparent dose-response effect. The pathways and correlates of harm underlying the increased risks of injury (particularly selfharm and assault) associated with marijuana use, require public health attention.

\section{ALCOHOL OUTLET DENSITY AND HOSPITAL ADMISSIONS FOR ALCOHOL-RELATED INJURY: AN ELECTRONIC RECORD-LINKED COHORT STUDY}

${ }^{1}$ Sarah Rodgers, ${ }^{2}$ Daniel Farewell, ${ }^{2}$ Frank Dunstan, ${ }^{2}$ James White, ${ }^{3}$ Scott Orford, ${ }^{2}$ Jennifer Morgan, ${ }^{1}$ Richard Fry, ${ }^{1}$ Ronan Lyons, ${ }^{2}$ David Fone. ${ }^{1}$ Farr Institute, Swansea University, UK; ${ }^{2}$ Institute of Primary Care and Public Health, Cardiff University, UK; ${ }^{3}$ School of Planning and Geography, Cardiff University, UK

\subsection{6/injuryprev-2016-042156.302}

Background Little is known on the longitudinal relationship between alcohol availability and the risk of admission to hospital for an alcohol-related injury. We aimed to quantify this relationship using observational record-linked data in a total adult population cohort in Wales, UK; population 2.5 million aged 16 years and over.

Methods Data sources included licensed outlets held by the 22 local authorities in Wales under The Licensing Act 2003 for each quarter between 2006 and 2011. Alcohol outlet density was estimated for Census geography small areas $(n=1896$ divided into quintiles of equal counts) based on the mean network walking distances between each household and alcohol outlet within a 10 minute walk. Hospital admissions 2006-2011 from the Patient Episode Database for Wales (PEDW) for wholly alcohol-related conditions were anonymously record-linked to the Welsh Demographic Service age-sex register within the Secure Anonymised Information Linkage Databank. Injury admissions were defined by ICD-10 codes S00-99 and T00-19 linked to an alcohol code. Longitudinal statistical analysis used Cox regression models of hospital admissions as a function of outlet density at baseline, adjusting for confounding variables of age, sex and small area deprivation and settlement type, and censoring for death, migration and other cause admission.

Results Alcohol outlet density at baseline was significantly associated with emergency hospital admission; the hazard ratio (HR) of an alcohol-related admission $(n=25,722)$ for living in the highest compared with the lowest quintile of outlet density was 1.17 (95\% CI: 1.11, 1.23). The risk was higher for alcohol-related admissions with injury ( $\mathrm{n}=4,308$; HR 1.27, 95\% CI: 1.13 , 1.42).

Conclusions Higher alcohol outlet availability was associated with more emergency hospital admissions for alcohol-related injury. This suggests that restricting the density of alcohol outlets within walking distance from home may improve population health.

\section{DOES INCREASING ALCOHOL TAXES ALWAYS REDUCE ALCOHOL-RELATED CRASHES: MARYLAND SALES TAX INCREASE?}

${ }^{1}$ Marie-Claude Lavoie, ${ }^{1}$ Gordon S Smith, ${ }^{1}$ Patricia Langenberg, ${ }^{2}$ Andres Villaveces, ${ }^{1}$ Patricia Dischinger, ${ }^{1}$ Linda Simoni-Wastila, ${ }^{1}$ Kathleen Hoke. ${ }^{1}$ University of Maryland, USA; ${ }^{2}$ World Bank, Washington, DC, USA

\subsection{6/injuryprev-2016-042156.303}

Background The sales tax on all alcoholic drink was raised in Maryland from 6 to 9\% on July 1, 2011. This study evaluates the effects of the alcohol sales tax increase on the rate of alcoholinvolved drivers involved in fatal crashes. 